\title{
ESTABILIDAD TÉRMICA DE ACEITE VIRGEN DE COCO OBTENIDO DE DOS CULTIVARES CRECIDOS EN GUERRERO, MÉXICO
}

\author{
THERMAL STABILITY OF VIRGIN COCONUT OIL OBTAINED FROM \\ TWO CULTIVARS GROWN IN GUERRERO, MEXICO
}

\section{Fabiola Elodio-Policarpo', Brenda Peñaloza-Herrera', Yanik I. Maldonado-Astudillo, 2 , Javier Jimenez-Hernandez ${ }^{1,2}$, Verónica Flores-Casamayor ${ }^{3}$, Gerónimo Arámbula-Villa ${ }^{3}$ y Ricardo Salazar ${ }^{4}$ *}

\begin{abstract}
'Universidad Autónoma de Guerrero, Facultad de Ciencias Químico Biológicas. Chilpancingo de los Bravo, Guerrero, México. ²Universidad Autónoma de Guerrero, Unidad de Estudios de Posgrado e Investigación. Acapulco, Guerrero, México ${ }^{3}$ Centro de Investigación y de Estudios Avanzados, Unidad Querétaro, Querétaro, México. ${ }^{4}$ CONACyT-Universidad Autónoma de Guerrero, Chilpancingo de los Bravo, Guerrero, México.
\end{abstract}

*Autor para correspondencia (rsalazarlo@conacyt.mx)

\section{RESUMEN}

El interés por el aceite virgen de coco ha crecido en los últimos años debido a sus efectos benéficos para la salud y a sus características fisicoquímicas y sensoriales atractivas para el consumidor. En este estudio se evaluó la estabilidad térmica del aceite virgen de coco extraido a partir de dos cultivares crecidos en el estado de Guerrero, México. Los resultados se compararon con un aceite de coco virgen comercial. Los aceites estudiados mostraron una composición similar de ácidos grasos. Se determinó el cambio en el contenido de ácidos grasos libres, fenoles y flavonoides totales, color, espectro de infrarrojo y valores de los coeficientes de extinción $\mathrm{K}_{232}$ y $\mathrm{K}_{270}$ de los aceites durante $5 \mathrm{~d}$ almacenados a 80,100 y $120^{\circ} \mathrm{C}$ en un horno de convección forzada. El contenido de compuestos fenólicos totales para el aceite virgen de coco comercial (AVCC), aceite virgen elaborado del cocotero Híbrido Mapan (AVCHM) y aceite virgen elaborado del cocotero Alto del Pacífico (AVCAP) fue $3.00 \pm 0.30,60.04 \pm 4.10$ y $78.00 \pm 6.20 \mathrm{mg}$ equivalentes de ácido gálico $100 \mathrm{~g}^{-1}$ aceite, respectivamente. Los AVCHM y AVCAP de Guerrero, México mostraron una estabilidad oxidativa significativamente mayor al AVCC ( $P$ $\leq 0.05$ ). Lo anterior se concluyó al no observar cambios importantes en el valor de índice de peróxidos, porcentaje de ácidos grasos, coeficientes de extinción $\mathrm{K}_{232}$ y $\mathrm{K}_{270}$ y ángulo matiz. La presencia de compuestos oxidados después del almacenamiento se demostró con espectroscopía infrarroja a través de los cambios en las frecuencias 1746 y $3470 \mathrm{~cm}^{-1}$ que corresponden a la presencia de compuestos carbonílicos e hidroperóxidos, respectivamente. Los resultados obtenidos en este estudio indican que los aceites de coco artesanales elaborados en Guerrero, México tienen un mayor potencial tecnológico por su elevado contenido de compuestos fenólicos y mayor estabilidad oxidativa en comparación con el aceite de coco comercial.

Palabras clave: Cocos nucifera, cocotero, estabilidad térmica, compuestos fenólicos.

\section{SUMMARY}

The interest in virgin coconut oil has grown in recent years due to its beneficial effects on health and its attractive physicochemical and sensorial characteristics for the consumer. In this study, the thermal stability of virgin oil coconut oil extracted from two cultivars grown in the state of Guerrero, Mexico was evaluated. The results were compared with a commercial virgin coconut oil. The studied oils showed a similar composition of fatty acids. The change in the content of free fatty acids, phenols and total flavonoids, color, infrared spectrum and values of the $\mathrm{K}_{232}$ and $\mathrm{K}_{270}$ extinction coefficients of the oils stored for $5 \mathrm{~d}$ at 80,100 and $120^{\circ} \mathrm{C}$ in a forced convection oven were determined. The content of total phenolic compounds for commercial virgin coconut oil (CVCO), virgin oil produced from the Mapan Hybrid coconut palm (VOMHC) and virgin oil from Alto del Pacifico coconut palm (VOAPC) was 3.00 $\pm 0.30,60.04 \pm 4.10$ and $78.00 \pm 6.20 \mathrm{mg}$ equivalents of gallic acid $100 \mathrm{~g}^{-1}$ oil, respectively. The VOMHC and VOAPC of Guerrero, Mexico showed significantly greater oxidative stability than the CVCO $(P \leq 0.05)$. The above was concluded as no important changes were observed in the value of peroxide index percentage of fatty acids, $\mathrm{K}_{232}$ and $\mathrm{K}_{270}$ extinction coefficients and hue angle. The presence of oxidized compounds after storage was demonstrated with infrared spectroscopy through the changes in frequencies 1746 and $3470 \mathrm{~cm}^{-1}$ corresponding to the presence of carbonyl compounds and hydroperoxides, respectively. The results obtained in this study indicate that artisanal coconut oils made in Guerrero, Mexico have a greater technological potential due to their high content of phenolic compounds and greater oxidative stability compared to commercial coconut oil.

Index words: Cocos nucifera, coconut palm, thermal stability, phenolic compounds

\section{INTRODUCCIÓN}

El interés por el aceite virgen de coco (AVC) ha crecido en los últimos años debido a sus efectos benéficos para la salud y a sus características fisicoquímicas y sensoriales, atractivas para el consumidor, con respecto a los atributos de otros aceites vegetales comestibles (Marina et al., 2009). El aceite de coco es ampliamente utilizado en la industria alimentaria, farmacéutica y de cosméticos. El AVC se define como el aceite obtenido de la pulpa fresca y madura de la semilla del cocotero (Cocus nucifera L.) por medios naturales o mecánicos, con o sin el uso de calor, no sometido a un tratamiento químico, refinado, blanqueo o desodorizado que altere la naturaleza del aceite (Villarino et al., 2007).

El cultivo del cocotero representa una actividad económica importante en las zonas costeras del estado de Guerrero. La producción de copra en el 2016 representó $11.1 \%$ del valor total de la producción agrícola del estado (SIAP, 2016). En los últimos años, la producción de AVC obtenido del endospermo o pulpa del coco ha constituido una alternativa para el incremento de los ingresos de los 
productores. Los principales cultivares utilizados para la extracción de aceite en Guerrero son los cocoteros Alto del Pacífico e Híbrido Mapan. Dichos cultivares se caracterizan por su resistencia al amarillamiento letal, precocidad y por su alta producción de copra y agua (Cortázar, 2011).

A pesar del evidente beneficio económico que representa la extracción de aceite de coco, los productores no consideran la influencia del cultivar en las características fisicoquímicas del AVC obtenido, lo que ocasiona una gran disparidad entre las características de calidad de los aceites producidos en la región, lo que afecta de forma negativa la competitividad en el sector. En este sentido, la estabilidad térmica del aceite, definida como la capacidad que presenta un compuesto de permanecer inalterado frente al calentamiento, es uno de los principales parámetros de calidad, debido a que determina las características de almacenamiento, transformación, aplicación y comercialización para su uso en la industria (Villarino et al., 2007).

Diversos estudios han demostrado la relación entre el contenido de antioxidantes, la composición de ácidos grasos saturados y el método de extracción con la estabilidad oxidativa del AVC (Henna-Lu y Tan, 2009; Seneviratne y Dissanayake, 2008; Srivastava y Semwal, 2015); por lo tanto, se espera que las propiedades y composición del aceite de coco varíen con el cultivar de cocotero empleado. Debido a la importancia que la estabilidad oxidativa representa para determinar el potencial tecnológico de los aceites elaborados en Guerrero, México, el presente estudio tuvo como objetivo evaluar la estabilidad térmica de aceite de coco virgen elaborado a partir de dos de los cultivares más importantes (Alto del Pacífico e Híbrido Mapan) del estado con la finalidad de identificar áreas de oportunidad para su comercialización.

\section{MATERIALES Y MÉTODOS}

\section{Material vegetal y área de procedencia}

Se utilizaron cocos de los cultivares de cocoteros Alto del Pacífico e Híbrido Mapan en la misma etapa de madurez (10 a 12 meses) para obtener el aceite virgen de coco (AVC); los cocos se recolectaron en el mes de septiembre del 2015 en el poblado de Chautengo, municipio de Florencio Villarreal (16 38' 34.1" latitud N, 99 05' 43.2" longitud 0, 8 msnm) en la región de Costa Chica del estado de Guerrero. Se deshidrataron parcialmente al sol $5 \mathrm{~kg}$ de pulpa de cada uno de los cultivares mencionados durante $72 \mathrm{~h}$. La pulpa de coco se rayó, se colocó en una cacerola de acero inoxidable y se calentó a una temperatura cercana a $100{ }^{\circ} \mathrm{C}$ para extraer el AVC durante $15 \mathrm{~min}$. El aceite de coco comercial de elaboración nacional se obtuvo de un supermercado de la ciudad de Chilpancingo, Guerrero. Todos los reactivos utilizados fueron grado analítico y se adquirieron en Sigma-Aldrich ${ }^{\circledR}$ (St. Louis, MO, EUA) O JT Baker® (Phillipsburg, NJ, EUA).

\section{Composición de ácidos grasos}

La composición de ácidos grasos de los aceites estudiados se determinó por cromatografía de gases, después de su derivatización a ésteres metílicos de ácidos grasos con $\mathrm{KOH} 2 \mathrm{~N}$ en metanol, de acuerdo con los métodos oficiales 2.301 y 2.302 de IUPAC (1992). Para este análisis se utilizó un cromatógrafo de gases marca Agilent modelo 7890B (Agilent Technologies, Santa Clara, California, EUA) equipado con una columna capilar Innowax (longitud $30 \mathrm{~m}$, diámetro interno $0.25 \mathrm{~mm}$, película $0.20 \mu \mathrm{m})(J \& C$ Scientific, Agilent Technologies, Pittsburgh, Pennsylvania, EUA) y un detector de ionización de flama. Como gas acarreador se utilizó hidrógeno $(1 \mathrm{~mL}$ $\left.\mathrm{min}^{-1}\right)$. La temperatura del detector y del inyector fue de $250{ }^{\circ} \mathrm{C}$. La temperatura inicial del horno fue de $60^{\circ} \mathrm{C}$, se mantuvo 5 min y después se incrementó con un gradiente de temperatura de $5^{\circ} \mathrm{C} \mathrm{min}{ }^{-1}$ hasta $200^{\circ} \mathrm{C}$. Posteriormente, se incrementó a $240{ }^{\circ} \mathrm{C}$ a $5{ }^{\circ} \mathrm{C}$ min-1 $^{-1}$ y se mantuvo así durante $7 \mathrm{~min}$. Para la identificación de los ácidos grasos se utilizó como estándar una mezcla de ésteres metílicos de ácidos grasos (C4-C24) (Supelcoß Inc.)

\section{Deterioro acelerado de los aceites}

Con la finalidad de acelerar las reacciones de deterioro tales como la oxidación, hidrólisis y polimerización de los aceites, el calentamiento se realizó a 80,100 y $120^{\circ} \mathrm{C}$ en un horno de convección forzada (Luzeren ${ }^{\circledR}$, DHG-9070A; Beijing, China) durante $5 \mathrm{~d}$. Las mediciones se realizaron cada $24 \mathrm{~h}$.

\section{Determinación del índice de peróxidos, porcentaje de ácidos grasos, coeficientes de extinción $\mathrm{K}_{232} \mathrm{y} \mathrm{K}_{270} \mathrm{y}$ color}

El índice de peróxidos (IP) y el porcentaje de ácidos grasos (\% AG) se calcularon de acuerdo con los métodos Cd 8-53 y Ca 5a-40 de la American Oil Chemists' Society (AOCS, 2003), respectivamente. Los coeficientes de extinción $\left(K_{232}\right.$ y $\left.K_{270}\right)$ de las muestras de aceite oxidadas se calcularon a partir de los valores de absorción medidos a una longitud de onda de 232 y $270 \mathrm{~nm}$, respectivamente. Los cálculos se realizaron con la ecuación $K_{\lambda}=D_{\lambda} C^{-1}$ donde $K_{\lambda}$ es el coeficiente de extinción para cada longitud de onda, $D_{\lambda}$ es la absorbancia y $\mathrm{C}$ es la concentración de aceite en $\mathrm{g} 100 \mathrm{~mL}^{-1}$. Se utilizó una celda de cuarzo de $1 \mathrm{~cm}$ de camino óptico (Paz y Molero, 2000). El color de las muestras de aceite se determinó con un colorímetro 
portátil (X-Rite Spectrophotometer Model Ci62, X-Rite Incorporated, Grandville, Michigan, EUA). El ángulo matiz $(H *)$ en los diferentes periodos de tiempo se calculó con los parámetros de la escala CIE $L * a * b *: H *=\tan ^{-1}(b * / a *)$, donde $\mathrm{a}^{*}=$ [negativo (verde) a positivo (rojo)], $\mathrm{b} *=$ [negativo (azul) a positivo (amarillo)].

\section{Determinación de los fenoles y flavonoides totales}

Los fenoles de las muestras de aceite se extrajeron siguiendo el procedimiento reportado por Henna-Lu y Tan (2009) modificado en el laboratorio. La cantidad de 0.5 $\mathrm{g}$ de aceite se disolvió en $5 \mathrm{~mL}$ de hexano con $5 \mathrm{~mL}$ de metanol:agua (60:40 v/v). La mezcla se agitó en un vórtex durante 5 min y se centrifugó a $2000 \times g$ por 5 min. La fase hidroalcohólica se colectó y se almacenó en refrigeración hasta su uso para la determinación de ambos parámetros. El contenido de fenoles totales se cuantificó a través del método de Folin-Ciocalteau. Ácido gálico (AG) se utilizó como estándar y los resultados se expresaron como mg equivalentes de $A G$ por $100 \mathrm{~g}$ de aceite. El contenido de flavonoides totales se determinó mediante el método colorimétrico de $\mathrm{AlCl}_{3}$ reportado por Adom y Liu (2002). Como estándar se usó catequina y los resultados se expresaron en $\mathrm{mg}$ equivalentes de catequina por $100 \mathrm{~g}$ de aceite.

\section{Espectroscopía infrarroja con transformada de Fourier (FTIR)}

Los espectros de infrarrojo se colectaron con la técnica de reflectancia difusa en un espectro de infrarrojo con transformada de Fourier marca Perkin-Elmer ${ }^{\circledR}$ Spectrum $^{\text {TM }}$ GX (Waltham, Massachusetts, EUA) en un rango de 400$4000 \mathrm{~cm}^{-1}$ con una resolución de $4 \mathrm{~cm}^{-1}$ y un número de barridos de 24 .

\section{Análisis estadístico}

Todos los resultados se expresaron como promedios (n = 3). Un análisis de varianza de una sola vía a través de un diseño de medias repetidas se utilizó para las cinéticas de calentamiento. Las diferencias entre los diferentes grupos se determinaron con la prueba de Tukey. Todos los análisis estadísticos se llevaron a cabo con el paquete JMP 9.0 de SAS en el nivel de significancia de $a=0.05$.

\section{RESULTADOS Y DISCUSIÓN}

\section{Composición de ácidos grasos}

La composición de ácidos grasos de los aceites estudiados (Cuadro 1) fue comparable con la sugerida en el Codex Alimentario (Codex, 2003); sin embargo, en el aceite virgen de coco comercial (AVCC) se detectaron pequeñas cantidades del ácido trans oleico (C18:1). Los ácidos grasos trans se forman generalmente durante el refinado de los aceites comestibles (Vaisali et al., 2015) y están asociados con un incremento en el riesgo de enfermedades cardiacas y de las arterias coronarias (Souza et al., 2015).

En las tres muestras analizadas, los ácidos mayoritarios correspondieron al ácido láurico (C12:0) y mirístico (C14:0).

Cuadro 1. Composición (\%) de ácidos grasos (\%) de los aceites estudiados.

\begin{tabular}{|c|c|c|c|c|}
\hline \multirow{2}{*}{ Nombre común } & \multirow{2}{*}{ Número lipídico } & \multicolumn{3}{|c|}{ Procedencia } \\
\hline & & Comercial & Híbrido Mapan & Alto del Pacífico \\
\hline Ácido caproico & C6:0 & $0.55 \mathrm{a}$ & $0.60 \mathrm{a}$ & $0.60 \mathrm{a}$ \\
\hline Ácido caprílico & C8:0 & $6.72 b$ & $7.11 \mathrm{ab}$ & $7.57 \mathrm{a}$ \\
\hline Ácido cáprico & C10:0 & $5.44 b$ & $5.57 b$ & $6.37 \mathrm{a}$ \\
\hline Ácido láurico & C12:0 & $44.79 \mathrm{c}$ & $46.60 \mathrm{~b}$ & $49.85 a$ \\
\hline Ácido mirístico & $\mathrm{C} 14: 0$ & $19.08 \mathrm{a}$ & $19.25 \mathrm{a}$ & $18.12 \mathrm{a}$ \\
\hline Ácido palmítico & $\mathrm{C} 16: 0$ & $9.37 \mathrm{ab}$ & $9.60 \mathrm{a}$ & $8.63 b$ \\
\hline Ácido esteárico & C18:0 & 3.08 a & $2.93 \mathrm{a}$ & $3.15 a$ \\
\hline Ácido oleico & C18:1 & $6.77 \mathrm{a}$ & $6.72 \mathrm{a}$ & $4.30 \mathrm{~b}$ \\
\hline Ácido trans oleico & C18:1 trans & 0.44 & nd & nd \\
\hline Ácido linoleico & C18:2 & $1.37 \mathrm{a}$ & $1.47 \mathrm{a}$ & $1.28 \mathrm{~b}$ \\
\hline Ácido linolénico & C18:3 & $0.05 a b$ & $0.07 \mathrm{a}$ & $0.02 b$ \\
\hline Ácido araquídico & $\mathrm{C} 20: 0$ & $0.55 a$ & $0.08 \mathrm{~b}$ & $0.09 \mathrm{~b}$ \\
\hline
\end{tabular}

Promedios seguidos de una letra diferente en la misma fila son significativamente diferentes; nd: no detectado. 
Los valores del ácido láurico se ubicaron entre 49.85 y 44.79 $\%$ pero su contenido en el AVCC fue significativamente menor. Estas desigualdades pueden estar relacionadas con las diferencias geográficas de la procedencia de los cocoteros y con los procesos de extracción utilizados. Por otra parte, el aceite virgen de coco Alto del Pacífico (AVCAP) mostró el mayor contenido de ácidos grasos de cadena media ácido caproico (C6:0), ácido caprílico (C8:0), ácido cáprico (C10:0) y ácido láurico (C12:0).

La mejora en la absorción de calcio y la disminución de los niveles de colesterol y triglicéridos en sangre se tiene reportada entre los diversos beneficios a la salud de los ácidos grasos de cadena media encontrados en el AVC, debido a que son digeridos, absorbidos y metabolizados de manera distinta que los ácidos grasos de cadena larga (Sáyago-Ayerdi et al., 2008). Diversos estudios señalan el efecto cardioprotector del aceite de coco (Babu et al., 2014; Eyres et al., 2016). En el mismo sentido, el AVCC y el AVCHM presentaron los mayores porcentajes de ácido oleico. No obstante los beneficios del ácido oleico a la salud, su presencia en los aceites vegetales está correlacionado directamente con la estabilidad oxidativa de los mismos (Frega et al., 1999).

\section{Contenido de compuestos fenólicos y flavoniodes}

El contenido de compuestos fenólicos fue de $3.00 \pm$

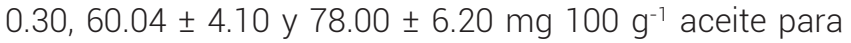
AVCC, AVCHM y AVCAP, respectivamente. El contenido de flavonoides totales fue de $88.68 \pm 2.55$ y $122.02 \pm 14.74$ mg $100 \mathrm{~g}^{-1}$ aceite para AVCHM y AVCAP, respectivamente. En el AVC comercial no se detectaron flavonoides. Estas diferencias pueden estar relacionadas con los diferentes métodos de extracción utilizados. Los aceites artesanales de este estudio se obtuvieron a partir de la cocción de pulpa de coco deshidratada (copra) durante un tiempo prolongado y una elevada temperatura. Durante este proceso los compuestos fenólicos presentes en la copra son incorporados en el aceite.

Generalmente, el aceite de coco comercial se prepara a partir del prensado de la copra, y el aceite es extraído rápidamente a baja temperatura. El aceite comercial posiblemente se somete a un proceso físico de refinado para proporcionarle un aspecto limpio y un color claro, lo que afecta el contenido de polifenoles. De acuerdo con Seneviratne et al. (2009), la elevada temperatura y el tiempo prolongado de exposición de la pulpa de coco para obtener AVC artesanales favorece un incremento en el contenido de compuestos fenólicos; asimismo, el contenido de compuestos fenólicos obtenidos en este estudio para los aceites originarios del estado de Guerrero, México fue aproximadamente el doble de los reportados para AVC comercializados en Malasia e Indonesia (Marina et al., 2009).

Independientemente de la temperatura y el AVC estudiado, el contenido de compuestos fenólicos disminuyó significativamente durante el calentamiento de los aceites. Después de $24 \mathrm{~h}$ a $80{ }^{\circ} \mathrm{C}$, el contenido inicial de compuestos fenólicos en AVCC se redujo $40 \%$ y a las $48 \mathrm{~h}$ no se detectó la presencia de estos compuestos. Por otro lado, para los aceites artesanales provenientes de los cultivares Híbrido Mapan y Alto del Pacífico, después de $5 \mathrm{~d}$ a $80{ }^{\circ} \mathrm{C}$, el contenido inicial de compuestos fenólicos se redujo 53.29 y $64.10 \%$, respectivamente. A 100 y 120 ${ }^{\circ} \mathrm{C}, \mathrm{AVCC}, \mathrm{AVCHM}$ y AVCAP mostraron una reducción de 40, $47,48 \%$ y $66,70,79 \%$ a las 24 h, respectivamente; pero 48 h después, no se detectó la presencia de compuestos fenólicos en los aceites estudiados. De manera análoga, a las $24 \mathrm{~h}$ y a las tres temperaturas estudiadas, no se detectaron flavonoides en AVCHM y AVCAP. Srivastava y Semwal (2015) mencionaron que, en general, tiempos prolongados y temperaturas elevadas favorecen la oxidación de los compuestos fenólicos presentes en el aceite de coco.

\section{Índice de peróxidos}

En la Figura 1 se muestra el índice de peróxido de los aceites en función del tiempo de calentamiento. El índice de peróxido (IP) es un método estándar para medir la concentración de peróxidos e hidroperóxidos formados en la etapa inicial de la oxidación de los lípidos y es utilizado como un indicador de la estabilidad oxidativa de los aceites (van de Voort et al., 1994). Teóricamente, el aceite de coco debe mostrar una velocidad lenta de oxidación debido a su elevado contenido de ácidos grasos saturados; sin embargo, los resultados mostraron que independientemente de la temperatura empleada, el calentamiento indujo en el AVCC un incremento significativamente mayor del IP. A $120^{\circ} \mathrm{C}$ se observó una disminución del IP en el día 4. Este fenómeno refleja la conversión de los hidroperóxidos a otros compuestos más reactivos como aldehídos, cetonas y lactonas (YahuacaJuárez et al. 2013), productos de oxidación secundaria. De acuerdo con el Codex Alimentarius (2003), el valor máximo de IP para aceites vírgenes es de 15 meq $\mathrm{O}_{2} \mathrm{~kg}^{-1}$ aceite. Los aceites con valores de IP mayores a los señalados por el Codex Alimentarius (2003) son inestables y se enrancian con facilidad. Los valores de peróxidos de los aceites estudiados se encontraron en los rangos de 1.98 a 3.98, 2.00 a 11.95 y 1.99 a 8.00 meq $\mathrm{O}_{2} \mathrm{~kg}^{-1}$ aceite para AVCC, AVCHP y AVCAP, respectivamente. Debe resaltarse que el IP de los aceites artesanales elaborados a partir de los cocoteros Alto del Pacífico e Híbrido Mapan estuvo por debajo de los límites sugeridos, independientemente de la 
temperatura de calentamiento utilizada.

\section{Porcentaje de ácidos grasos en función del tiempo de calentamiento}

El porcentaje de ácidos grasos (\% AG) en función del tiempo de calentamiento de los aceites se muestra en la Figura 2 ejemplificado mediante el ácido láurico. Los ácidos grasos libres son los responsables de la aparición de aromas y sabores indeseables en los aceites, debido a lo cual, el \% AG es un buen indicador de la hidrólisis de los triacilglicéridos, ocasionada por acción enzimática o por la humedad, así como por la oxidación de los ácidos grasos insaturados presentes en el aceite. Inicialmente, el \% AG fue significativamente menor en el AVCC $(0.17 \%$ de ácido láurico), comparado con el AVCHM (0.35\% de ácido láurico) y con el AVCAP (0.30\% de ácido láurico). Los valores obtenidos fueron mayores a los reportados por
Marina et al. (2009). A $80^{\circ} \mathrm{C}$ no se observaron diferencias significativas entre el valor inicial de \% AG de los aceites estudiados. Por el contrario, a 100 y $120{ }^{\circ} \mathrm{C}$, el \% AG del AVCC se incrementó significativamente en relación con los aceites artesanales. Srivastava y Semwal (2015) registran un incremento de 8.9 veces en el valor de \% AG de AVC sometido a un proceso de freído durante $8 \mathrm{~h}$ a $180^{\circ} \mathrm{C}$.

\section{Coeficientes de extinción $\mathrm{K}_{232}$ y $\mathrm{K}_{270}$}

Los coeficientes de extinción $K_{232}$ (Figura 3 ) y $K_{270}$ (Figura 4) son un indicador de la presencia de dienos conjugados y compuestos carbonilos, respectivamente (Malheiro et al., 2009). Los valores del IP y de $K_{232}$ representan la presencia de compuestos de oxidación primaria (hidroperóxidos). Los dienos conjugados representados por el valor de $\mathrm{K}_{232}$ se forman a partir de los hidroperóxidos derivados de los ácidos grasos insaturados presentes en el aceite.
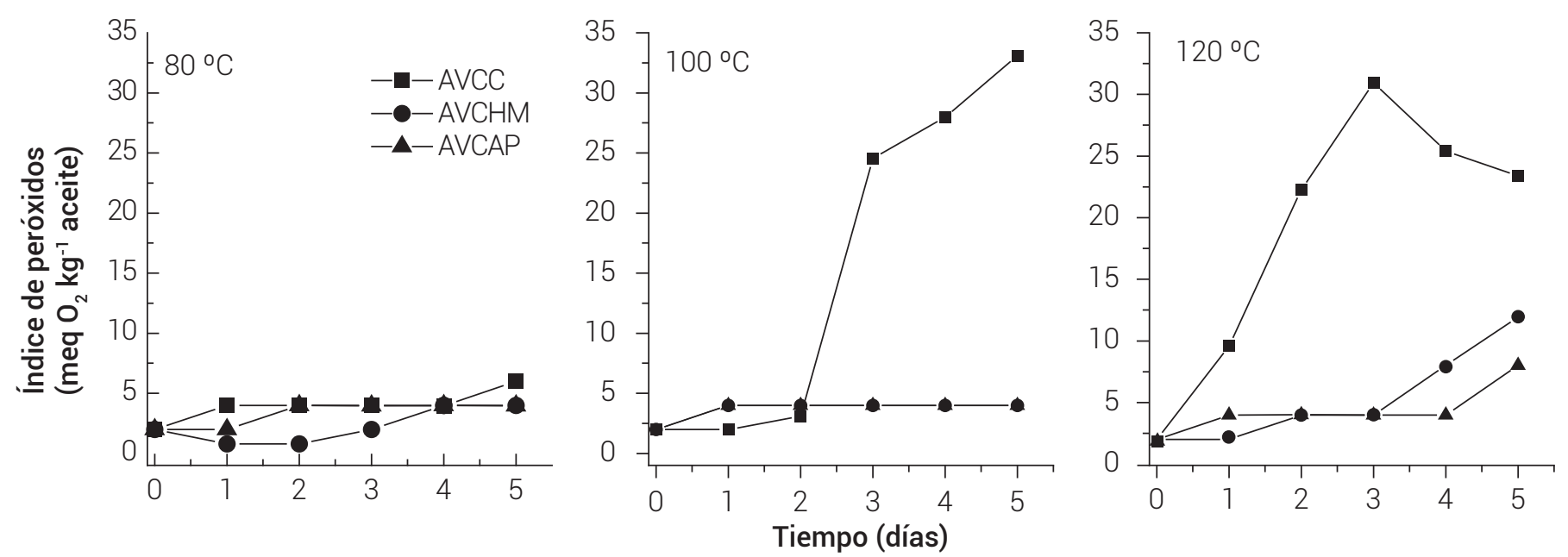

Figura 1. Variación del índice de peróxidos bajo tres temperaturas en función del tiempo de calentamiento en los aceites bajo estudio.
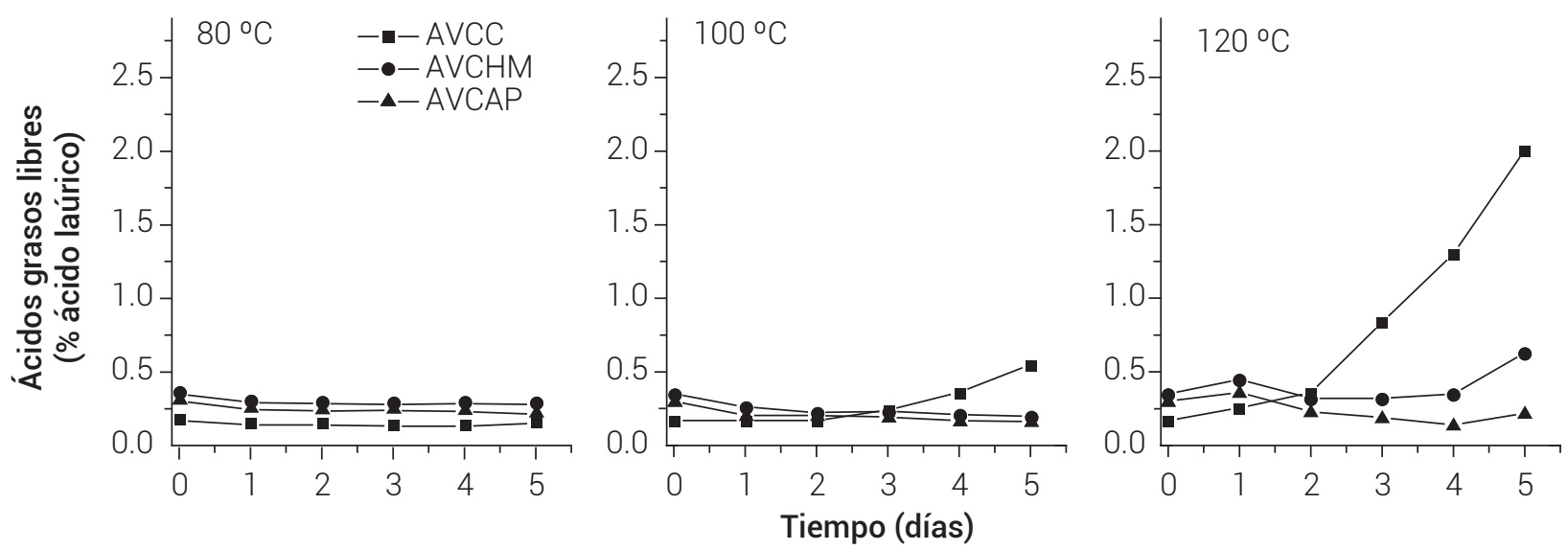

Figura 2. Variación del porcentaje de ácidos grasos libres a tres temperaturas en función del tiempo de calentamiento en los aceites bajo estudio. 
Por otro lado, el valor de $\mathrm{K}_{270}$ representa los compuestos de oxidación secundaria formados a partir de los hidroperóxidos antes mencionados.

Como se esperaba, se observó un comportamiento similar entre el IP y los valores de $\mathrm{K}_{232}$. A $80{ }^{\circ} \mathrm{C}$ no se observaron cambios importantes entre los aceites estudiados; por el contrario, para el AVCC, a 100 y $120^{\circ} \mathrm{C}$, en los días tres y uno, respectivamente, se observó un incremento significativo de los valores del coeficiente $\mathrm{K}_{232}$.

Debido a que la degradación de los compuestos de oxidación primarios produce compuestos de oxidación secundaria, se espera una relación inversa entre estos dos tipos de compuestos. En este sentido, los valores elevados de $\mathrm{K}_{270}$ obtenidos para el AVCC a 100 y $120{ }^{\circ} \mathrm{C}$ indican una mayor cantidad de productos de oxidación secundaria producidos de los hidroperóxidos formados a partir de la degradación de los ácidos insaturados presentes en el aceite. Es interesante hacer notar que los valores de $\mathrm{K}_{270}$ de AVCHM y AVCAP disminuyeron, independientemente de la temperatura, en el periodo de tiempo estudiado. Lo anterior sugiere que los compuestos de oxidación secundaria iniciales presentes en los aceites se degradaron a compuestos de bajo peso molecular que reaccionaron con otros grupos acilo para formar dímeros, oligómeros o polímeros (Choe y Min, 2006).

\section{Análisis de espectroscopía infrarroja}

El análisis de espectroscopía infrarroja por transformada de Fourier (FTIR) se utilizó para seguir la oxidación de los aceites durante el calentamiento. El análisis de FTIR tiene ventajas sobre los parámetros clásicos que se emplean para medir la estabilidad oxidativa de los aceites. En la caracterización de aceites vegetales las principales
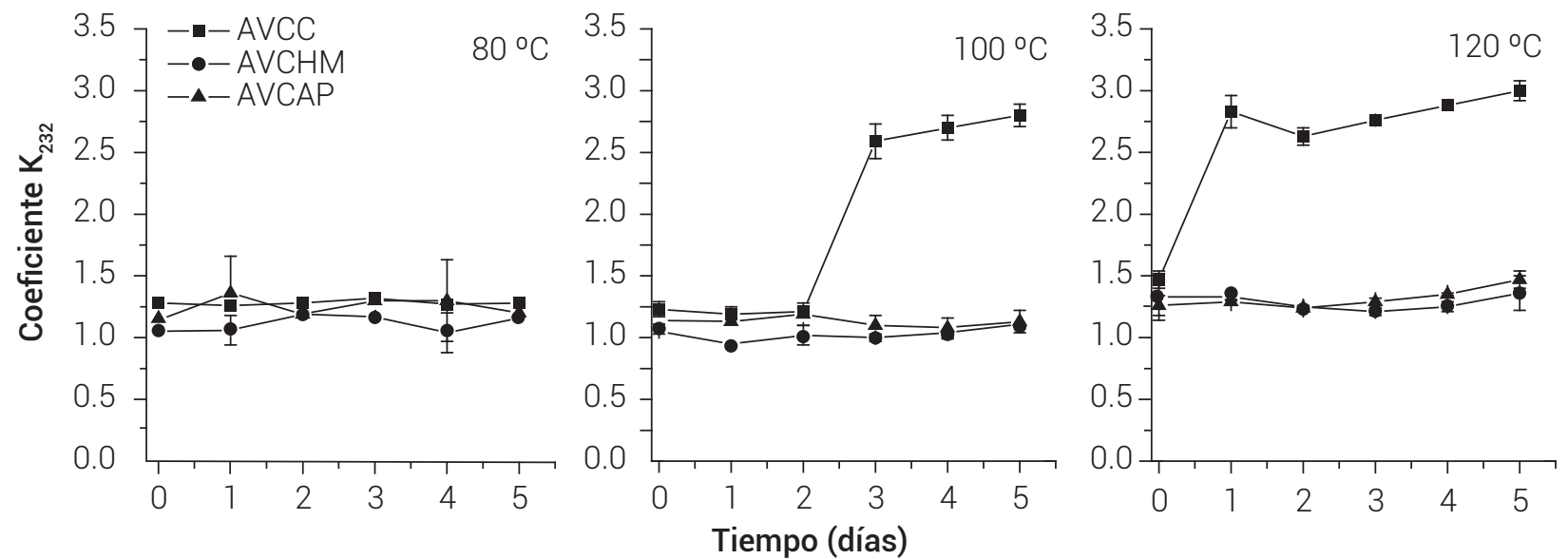

Figura 3. Variación del coeficiente de extinción $\mathrm{K}_{232}$ bajo tres temperaturas en función del tiempo de calentamiento en los aceites bajo estudio.
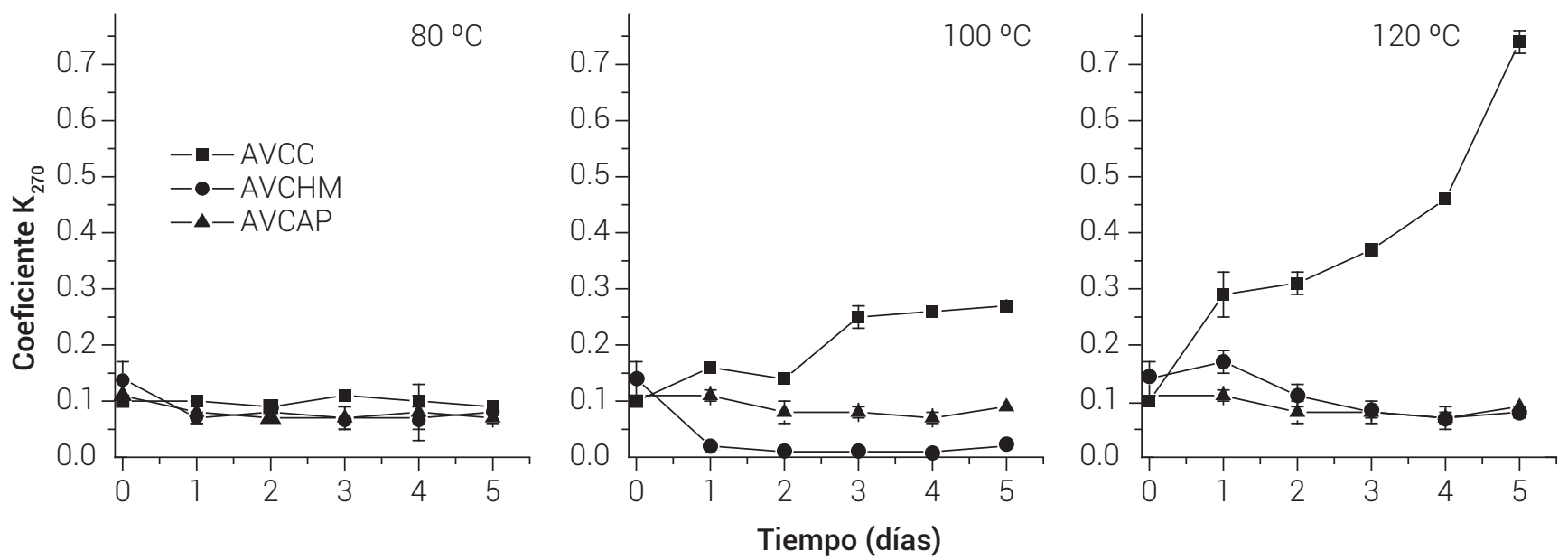

Figura 4. Variación del coeficiente de extinción $\mathrm{K}_{270}$ bajo tres temperaturas en función del tiempo de calentamiento en los aceites bajo estudio 
bandas de interés son los picos de reflectancia de 3600 a 3400 y 1800 a $1700 \mathrm{~cm}^{-1}$, las cuales corresponden a la presencia de hidroperóxidos y carbonilos, respectivamente (Rohman, 2017). En la Figura 5 se muestran los espectros de infrarrojo en el tiempo inicial y final de calentamiento de los aceites estudiados. El análisis visual de los espectros de los AVC a $80{ }^{\circ} \mathrm{C}$ no reveló diferencias apreciables con respecto al tiempo de calentamiento en los aceites artesanales; mientras que para el AVCC se observó una disminución en la intensidad de $1746 \mathrm{~cm}^{-1}$ y la presencia de una banda a $3550 \mathrm{~cm}^{-1}$, relacionada con la presencia de compuestos de oxidación secundaria como alcoholes (van de Voort et al., 1994), lo que a su vez indica la presencia de compuestos oxidados en el AVCC antes del calentamiento.

Las muestras calentadas durante $5 \mathrm{da} 120^{\circ} \mathrm{C}$ mostraron un aumento en las bandas a 1746 y $3470 \mathrm{~cm}^{-1}$, con excepción de AVCAP. Resultados similares se observaron a $100{ }^{\circ} \mathrm{C}$ (datos no mostrados). Como se mencionó anteriormente, la oxidación de los aceites involucra la formación de hidroperóxidos y dienos conjugados.
Estos compuestos evolucionan para formar productos de oxidación resultantes de la fragmentación de los triacilglicéridos, que producen compuestos de bajo y alto peso molecular (Guillen y Goicoechea, 2009). Durante el proceso de oxidación, la intensidad de la banda correspondiente a los hidroperóxidos $(\approx 3470$ $\mathrm{cm}^{-1}$ ) aumenta de forma proporcional a la formación de éstos. En las etapas más avanzadas de oxidación aparece una banda nueva a $3550 \mathrm{~cm}^{-1}$ que refleja la formación de alcoholes (Yahuaca-Juárez et al., 2013). En el mismo sentido, Smith et al. (2007) señalan que la descomposición de los hidroperóxidos forma compuestos con grupos carbonilos que se reflejan en altas intensidades de la banda encontrada a $1746 \mathrm{~cm}^{-1}$. La mayor estabilidad oxidativa observada en el AVCAP se puede atribuir a su mayor contenido de compuestos fenólicos entre los aceites estudiados. Wagner et al. (2001) demostraron que la presencia de antioxidantes incrementa la estabilidad oxidativa de aceite de coco durante el freído y el almacenamiento.
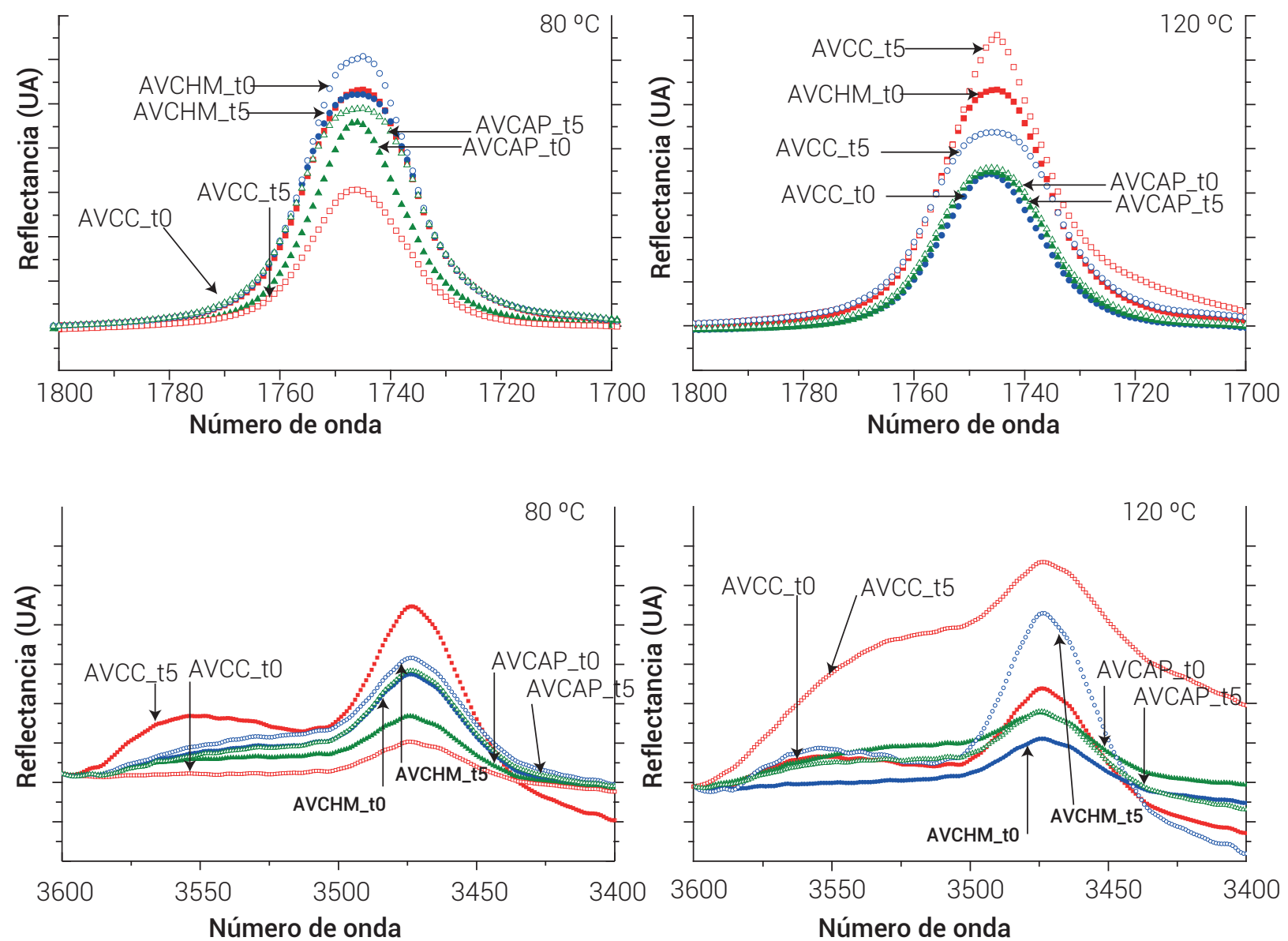

Figura 5. Espectros de infrarrojo en el tiempo inicial y final de calentamiento bajo dos tmperaturas de los aceites estudiados. 


\section{Cambios de color}

Los cambios de color de los aceites durante el calentamiento a $120^{\circ} \mathrm{C}$, representados por el ángulo matiz, se muestran en la Figura 6. El color es un indicador visual del deterioro de los aceites a consecuencia de su oxidación. Morelló et al. (2004) concluyeron que el oscurecimiento del aceite de oliva durante el almacenamiento es consecuencia de la degradación de los ácidos grasos insaturados y de los compuestos fenólicos presentes en los aceites. Los valores iniciales de ángulo matiz sugieren que los aceites mostraron un color amarillo, con un tono más claro para el AVCC seguido del AVCHM y AVCAP. Cabe mencionar que la intensidad de color en los aceites artesanales está relacionada con el mayor contenido de compuestos fenólicos en los mismos. Los AVCHM y AVCAP no mostraron diferencias significativas en el ángulo matiz durante su calentamiento a $120^{\circ} \mathrm{C}$. Resultados similares se obtuvieron a 80 y $100{ }^{\circ} \mathrm{C}$ (datos no mostrados); por el contrario, un incremento exponencial en color, hacia un amarillo intenso se observó para el AVCC en función del tiempo de exposición. El cambio en el ángulo matiz se atribuyó a la presencia de compuestos no volátiles derivados de la degradación oxidativa del aceite comercial.

Algunas de las implicaciones del contenido elevado de compuestos fenólicos de los aceites artesanales sugieren que éstos pueden ser empleados como una fuente natural de antioxidantes para detonar su potencial y expandir su presencia en el mercado. Aunque es necesario un análisis más detallado de la composición de los aceites, la estabilidad oxidativa de los aceites estudiados en el presente trabajo proporciona información valiosa a la industria sobre el tipo de aceite de coco más recomendable para obtener mejores productos en un espectro amplio de condiciones de procesamiento.

\section{CONCLUSIONES}

Los aceites de coco artesanales elaborados a partir de cocoteros Híbrido Mapan y Alto del Pacífico cultivados en el estado de Guerrero tienen una mayor estabilidad oxidativa que el aceite virgen de coco comercial. La estabilidad de los aceites se relacionó con los valores de $I P, \% A G$, coeficientes de extinción $K_{232}, K_{270} y$ ángulo matiz. La presencia de compuestos oxidados se demostró con espectroscopía infrarroja. El contenido de compuestos fenólicos en los aceites de coco artesanales fue elevado, pero con el incremento en la temperatura y el tiempo de calentamiento los compuestos fenólicos en el aceite se degradaron.

\section{BIBLIOGRAFÍA}

Adom K. K. and R. H. Liu (2002) Antioxidant activity of grains. Journal of Agricultural and Food Chemistry 50:6182-6187, https://doi. org/10.1021/jf0205099

AOCS, American Oil Chemists' Society (2003) Official Methods and Recommended Practices of the AOCS. Analytical Methods. D. Firestone (ed.). AOCS Press, Urbana, Illinois, USA. 1200 p.

Babu A. S., S. K. Veluswamy, R. Arena, M. Guazzi and C. J. Lavie (2014) Virgin coconut oil and its potential cardioprotective effects. Postgraduate Medicine 126:76-83. https://doi.org/10.3810/ pgm.2014.11.2835

Choe E. and D. B. Min (2006) Mechanisms and factors for edible oil oxidation. Comprehensive Reviews in Food Science and Food Safety 5:169-186, https://doi.org/10.1111/j.15414337.2006.00009.x

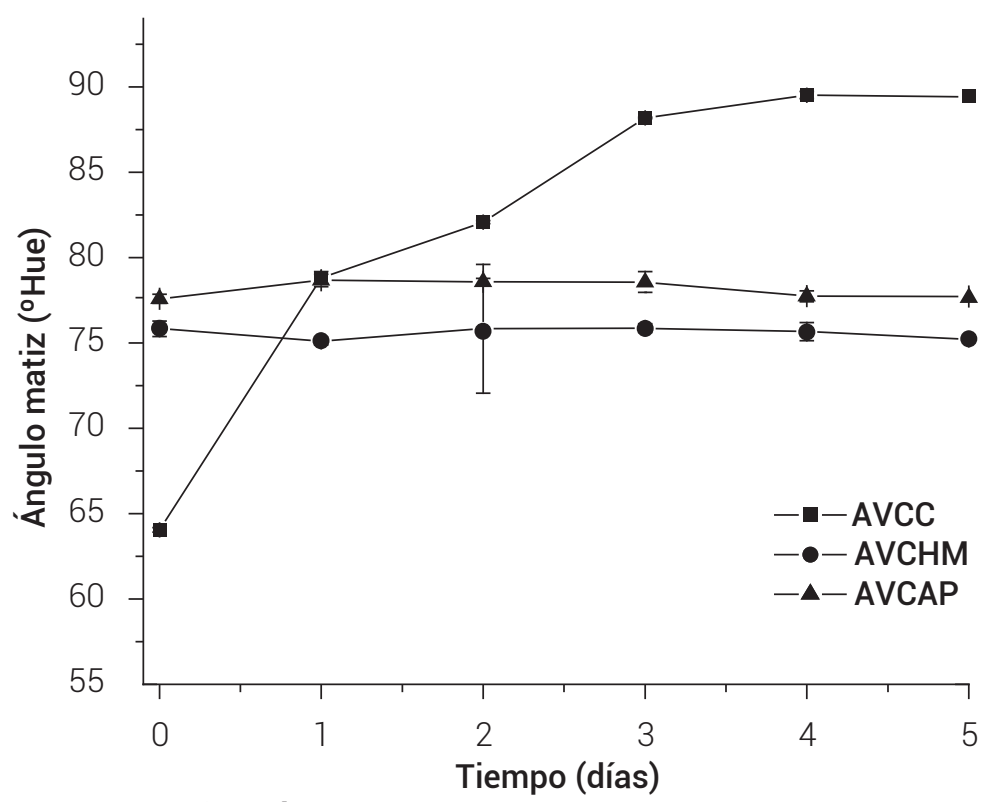

Figura 6. Variación del ángulo matiz en función del tiempo de calentamiento a $120^{\circ} \mathrm{C}$ en los aceites estudiados. 
Codex Alimentarius (2003) Standard for Named Vegetable Oils. Codex Stan 210-1999. Food and Agriculture Organization of the United Nations and World Health Organization. Geneva, Switzerland. $13 p$

Cortázar R. M. (2011) Paquete tecnológico palma de coco Alto Pacífico. Establecimiento y mantenimiento. Campo Experimental Chetumal, INIFAP. Chetumal, Quintana Roo. México. 12 p.

Eyres L., M. F. Eyres, A. Chisholm and R. C. Brown (2016) Coconut oil consumption and cardiovascular risk factors in humans. Nutrition Reviews 74:267-280, https://doi.org/10.1093/nutrit/ nuw002

Frega N., M. Mozzon and G. Lercker (1999) Effects of free fatty acids on oxidative stability of vegetable oil. Journal of the American Oil Chemists' Society 76:325-329, https://doi.org/10.1007/ s11746-999-0239-4

Guillen M. D. and E. Goicoechea (2009) Oxidation of corn oil at room temperature: primary and secondary oxidation products and determination of their concentration in the oil liquid matrix from 'H nuclear magnetic resonance data. Food Chemistry 116:183192, https://doi.org/10.1016/j.foodchem.2009.02.029

Henna Lu F. S. and P. P. Tan (2009) A comparative study of storage stability in virgin coconut oil and extra virgin olive oil upon thermal treatment. International Food Research Journal 16:343-354.

IUPAC, International Union of Pure and Applied Chemistry (1992) Standard Methods for the Analysis of Oils, Fats and Derivatives. $1^{\text {st }}$ Supplement to 7th edition. International Union of Pure and Applied Chemistry. Commission on Oils, fats and Derivatives. Blackwell Scientific Publications. Oxford, UK. 151 p.

Malheiro R., I. Oliveira, M. Vilas-Boas, S. Falcão, A. Bento and J. A. Pereira (2009) Effect of microwave heating with different exposure times on physical and chemical parameters of olive oil. Food and Chemical Toxicology 47:92-97, https://doi.org/10.1016/j. fct.2008.10.014

Marina A. M., Y. B. Che Man, S. A. H. Nazimah and I. Amin (2009) Chemical properties of virgin coconut oil. Journal of the American Oil Chemists' Society 86:301-307, https://doi.org/10.1007/ s11746-009-1351-1

Morelló J. R., M. J. Motilva, M. J. Tovar and M. P. Romero (2004) Changes in commercial virgin olive oil (cv Arbequina) during storage, with special emphasis on the phenolic fraction. Food Chemistry 85:357-364, https://doi.org/10.1016/j.foodchem.2003.07.012

Paz A. I. y M. Molero M. (2000) Aplicación de la espectrofotometría UVvisible al estudio de la estabilidad térmica de aceites vegetales comestibles. Grasas y Aceites 51:424-428.

Rohman A. (2017) Infrared spectroscopy for quantitative analysis and oil parameters of olive oil and virgin coconut oil: a review. International Journal of Food Properties 20:1447-1456, https:// doi.org/10.1080/10942912.2016.1213742

Sáyago-Ayerdi S. G., M. P. Vaquero, A. Schultz-Moreira, S. Bastida, and F. J. Sánchez-Muniz (2008) Utilidad y controversias del consumo de ácidos grasos de cadena media sobre el metabolismo lipoproteico y obesidad. Nutrición Hospitalaria 23:191-202.
Seneviratne K. N. and D. M. S. Dissanayake (2008) Variation of phenolic content in coconut oil extracted by two conventional methods International Journal of Food Science and Technology 43:597602, https://doi.org/10.1111/j.1365-2621.2006.01493.x

Seneviratne K. N., C. D. Hapuarachchl and S. Ekanayake (2009) Comparison of the phenolic-dependent antioxidant properties of coconut oil extracted under cold and hot conditions. Food Chemistry 114:1444-1449, https://doi.org/10.1016/j. foodchem.2008.11.038

SIAP, Servicio de Información Agroalimentaria y Pesquera (2016) Guerrero. Infografía Agroalimentaria 2016. Servicio de Información Agroalimentaria y Pesquera, SAGARPA. México, D, F. 51 p.

Smith S. A., R. E. King and D. B. Min (2007) Oxidative and thermal stabilities of genetically modified high oleic sunflower oil. Food Chemistry 102:1208-1213, https://doi.org/10.1016/j. foodchem.2006.06.058

Srivastava Y. and A. D. Semwal (2015) A study on monitoring of frying performance and oxidative stability of virgin coconut oil (VCO) during continuous/prolonged deep fat frying process using chemical and FTIR spectroscopy. Journal of Food Science and Technology 52:984-991, https://doi.org/10.1007/s13197-0131078-8

Souza R. J., A. Mente, A. Maroleanu, A. I. Cozma, V. Ha, T. Kishibe, E. Uleryk, P. Budylowski, H. Schünemann, J. Beyene and S. S. Anand (2015) Intake of saturated and trans unsaturated fatty acids and risk of all cause mortality, cardiovascular disease, and type 2 diabetes: systematic review and meta-analysis of observational studies British Medical Journal 351:h3978, https://doi.org/10.1136/ bmj.h3978

Vaisali C., S. Charanyaa, P. D. Belur and I. Regupathi (2015) Refining of edible oils: a critical appraisal of current and potential technologies International Journal of Food Science \& Technology 50:13-23, https://doi.org/10.1111/ijfs. 12657

van de Voort F. R., A. A. Ismail, J. Sedman, J. Dubois and T. Nicodemo (1994) The determination of peroxide value by Fourier transform infrared spectroscopy. Journal of the American Oil Chemists Society 71:921-926, https://doi.org/10.1007/BF02542254

Villarino B. J., L. M. Dy and M. C. C. Lizada (2007) Descriptive sensory evaluation of virgin coconut oil and refined, bleached and deodorized coconut oil. LWT - Food Science and Technology 40:193-199, https://doi.org/10.1016/j.lwt.2005.11.007

Wagner K. H., F. Wotruba and I. Elmadfa (2001) Antioxidative potentia of tocotrienols and tocopherols in coconut fat at different oxidation temperatures. European Journal of Lipid Science and Technology 103:746-751, https://doi.org/10.1002/14389312(200111)103:11<746::AID-EJLT746>3.0.CO;2-P

Yahuaca-Juárez B., H. E. Martínez-Flores, J. A. Huerta-Ruelas, R. C. Pless, P. A. Vázquez-Landaverde and R. Tello-Santillán (2013) Oil oxidation in corn flour from grains processed with alkaline cooking by use of peroxide value, UV and FTIR. Plant Foods for Human Nutrition 68:65-71, https://doi.org/10.1007/s11130-012-0332-y 
